\title{
Equilibrium Abundances of Heavy Elements Supported by Radiative Levitation in the Atmospheres of Hot DA White Dwarfs
}

\author{
P. CHAYER, ${ }^{1}$ S. VENNES, ${ }^{1}$ A. K. PRADHAN, ${ }^{2}$ P. THEJLL, ${ }^{3}$ \\ A. BEAUCHAMP, ${ }^{4}$ G. FONTAINE, ${ }^{4}$ AND F. WESEMAEL ${ }^{4}$ \\ ${ }^{1}$ Center for EUV Astrophysics, 2150 Kittredge St., University of California at Berkeley, \\ Berkeley, CA $94720-5030$, USA \\ ${ }^{2}$ Department of Astronomy, Ohio State University, Columbus, OH 43210-1106, USA \\ ${ }^{3}$ Niels Bohr Institute, Blegdamsvej 17, DK-2100, København $\varnothing$, Denmark \\ ${ }^{4}$ Département de Physique, Université de Montréal, C.P. 6128, Succ. Centre-Ville, \\ Montréal, Québec, Canada, H3C 3J7
}

\begin{abstract}
We present revised estimates of the equilibrium abundances of heavy elements supported by radiative levitation in the atmospheres of hot DA white dwarfs. We emphasize, in particular, the role of trace pollutants that may be present in the background plasma, an effect which has been heretofore neglected. We take advantage of the availability of a table of detailed monochromatic opacities calculated for a plasma made of $\mathrm{H}$ containing small amounts of $\mathrm{C}, \mathrm{N}, \mathrm{O}$, and $\mathrm{Fe}$ to illustrate how the equilibrium abundances of levitating elements react to the flux redistribution caused by the addition of these small traces of opaque material. We also consider two other improvements: a more sophisticated treatment of the momentum redistribution process and ion experiences following a photoexcitation, and use of an upgraded value for the line profile width associated with pressure broadening.
\end{abstract}

\section{Introduction}

The presence of pollutants in the high-gravity atmospheres of hot white dwarfs has received considerable attention in the last several years, thanks to important discoveries made in the UV, far ultraviolet (FUV), and extreme ultraviolet (EUV) spectral ranges. On the basis of such observations, quantitative abundance analyses have revealed, so far, the presence of $\mathrm{C}, \mathrm{N}, \mathrm{O}, \mathrm{Si}, \mathrm{Fe}$, and $\mathrm{Ni}$ in a handful of bright hot white dwarfs. To understand better how radiative levitation could potentially account for these and other possible atmospheric contaminants, Chayer, Fontaine, \& Wesemael (1995; hereafter referred to as CFW) recently presented the results of detailed calculations of radiative accelerations and equilibrium abundances for several elements in the envelopes of hot DA and DO/DB white dwarf models. This set of computations uses equilibrium surface abundances derived under the assumption of a strict balance between the radiative acceleration on a given element and the effective gravity at the Rosseland photosphere. This equilibrium approach, which ignores the potential influence of other competing mechanisms (e.g., stellar winds or accretion), constitutes but the first step toward understanding the observed patterns of trace heavy elements in hot white dwarfs.

CFW (see also Chayer et al. 1994) have pointed out that the most significant aspect of this equilibrium theory, which remains to be addressed in the white dwarf context, is the problem of flux blocking (or, more properly, flux redistribution) resulting from the simultaneous presence of traces of several different heavy elements in the atmospheres of hot stars. In order to assess the importance of this effect, we have carried out exploratory calculations in DA model atmospheres based on a representative mixture of $\mathrm{C}, \mathrm{N}, \mathrm{O}$, and $\mathrm{Fe}$ that, together with the dominant element ( $\mathrm{H}$ here), may provide a more realistic 
background monochromatic opacity than that provided by $\mathrm{H}$ alone. We further upgrade our approach to equilibrium radiative levitation theory in white dwarfs by combining the model atmosphere description with an implementation of the momentum redistribution process recently discussed by Gonzalez et al. (1995). As mentioned by CFW (their $\S$ 4), these considerations are the last remaining major improvements that need to be tackled in the framework of equilibrium radiative levitation theory.

\section{A Model Atmosphere Approach}

\subsection{Pure Hydrogen Models}

The computation of the total radiative acceleration on a given trace element is still based on equation (1) of CFW, except that the background monochromatic Eddington flux, $H_{\nu}$ (b), is evaluated through the full solution of the radiative transfer problem in the atmospheric layers instead of relying on approximations only formally valid at large optical depths. This approach is similar to the one followed by Bergeron et al. (1988) in the context of hot B subdwarfs. We compute the equilibrium abundances of levitating elements at the Rosseland photosphere $\left(\tau_{R}=2 / 3\right)$ of our model atmospheres, as these abundances are representative of the observable values. In order to provide a comparison point with the results of $\mathrm{CFW}$ as well as with the results of more complicated models below, we first consider the case of pure $\mathrm{H}$ atmosphere models.

The result is shown in Figure 1 where the expected equilibrium surface abundances of levitating elements are shown as functions of the effective temperature for pure $\mathrm{H}$ DA models which have $\log g=7.5$ and $T_{\text {eff }}=52,000,56,000,60,000$, and $64,000 \mathrm{~K}$. The solid curves show the results of CFW, while the dashed curves correspond to the improved results obtained with pure hydrogen model atmospheres. With one particularly outstanding exception, the predicted abundances of the two different approaches generally agree well within $0.3 \mathrm{dex}$. The outstanding exception occurs when the physical conditions are such that a closed-shell electronic configuration dominates the ionization balance.

\subsection{Hydrogen-Rich Models with Trace Heavy Elements}

The representative chemical composition of the background plasma we consider consists of a H-dominated mixture with small traces (by number) of carbon $\left(\mathrm{C} / \mathrm{H}=4 \times 10^{-7}\right)$, nitrogen $\left(\mathrm{N} / \mathrm{H}=5 \times 10^{-6}\right)$, oxygen $\left(\mathrm{O} / \mathrm{H}=10^{-6}\right)$, and iron $\left(\mathrm{Fe} / \mathrm{H}=10^{-5}\right)$. The $\mathrm{C}$ and $\mathrm{N}$ abundances have been determined by Wesemael, Henry, \& Shipman (1984), the O abundance by Chayer \& Vennes (1995), and the Fe abundance by Vennes et al. (1992) and Werner \& Dreizler (1994). These abundances are representative of the DA Feige 24.

The opacity tables were originally constructed to compute a small grid of model atmospheres suitable for the analysis of the hot DA star Feige 24 and its siblings (G191-B2B, RE 2214-492, and RE 0623-377), all of which have comparable atmospheric parameters. They are LTE models, and include a detailed treatment of blanketing effects through the use of some 30,000 frequency points. This choice was made on purpose in order to oversample the monochromatic opacity given in the tables. The models were computed by Vennes, Pradhan, \& Thejll (1995). They will be referred to as the HZ models in what follows.

We compare, in Figure 1, the predicted atmospheric abundances of our twelve standard heavy elements as functions of the effective temperature of the $\mathrm{HZ}$ (long dashed curves) and $H$ (dashed curves) atmosphere models. The expected equilibrium abundances of $\mathrm{S}, \mathrm{Ar}$, and $\mathrm{Ca}$ are larger, while those of $\mathrm{Na}, \mathrm{Mg}, \mathrm{Al}$, and $\mathrm{Fe}$ are smaller in presence of a contaminated background plasma compared to the pure $H$ case. Likewise, in both 


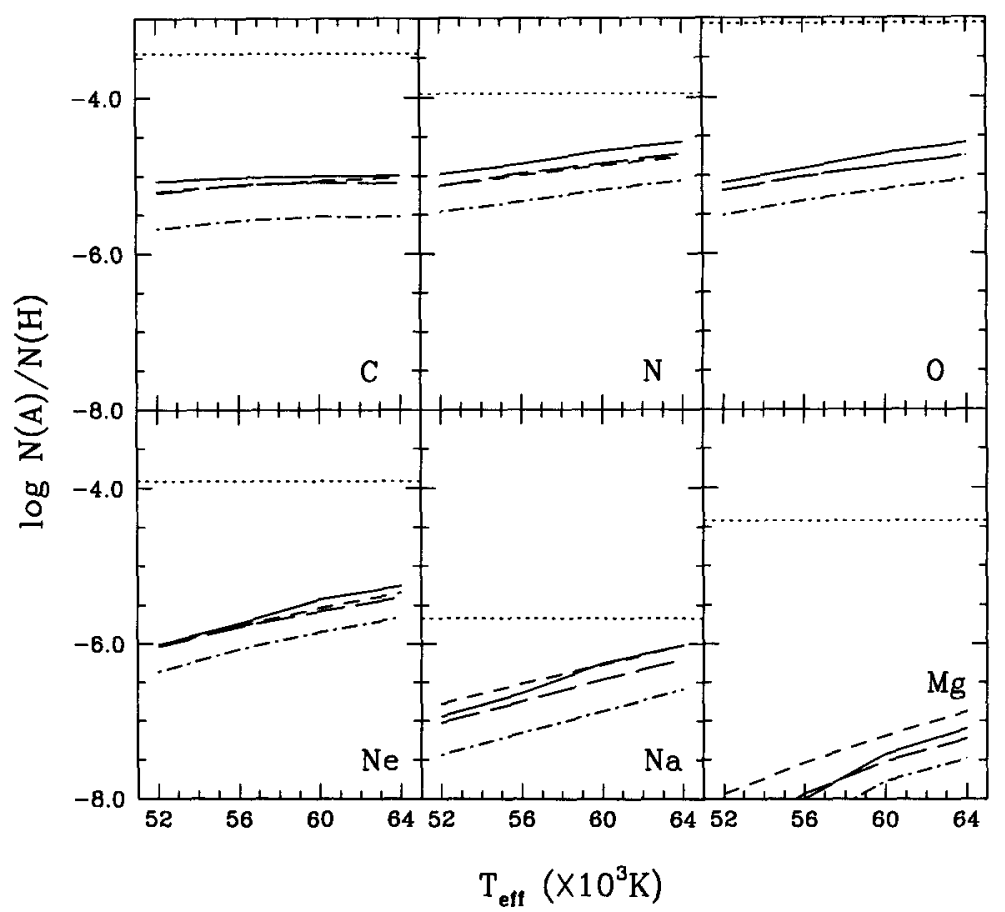

FIGURE 1. Expected equilibrium abundances of heavy elements levitating at the surface of hot DA white dwarfs. The equilibrium abundances are given with respect to that of hydrogen by number. The models have $\log g=7.5$. The solid curves show the results of CFW; the dashed curves show the results obtained with pure hydrogen model atmospheres; the long dashed curves correspond to the $\mathrm{HZ}$ models; the dot-dashed curves correspond to the $\mathrm{HZ}$ models but incorporate the improvements suggested by Gonzalez et al. (1995). The horizontal dotted line in each panel gives the cosmic abundance of the element of interest.

approaches, the abundances of $\mathrm{Ne}$ are comparable for the two kinds of background. For the levitating elements that do not belong to the background mixture (i.e., those other than $\mathrm{C}, \mathrm{N}, \mathrm{O}$, and $\mathrm{Fe}$ ), and except for possible accidental occurrences, the narrow absorption lines generally fall in the continuum of the energy distribution of an $\mathrm{HZ}$ model. For the needs of the present discussion, this "continuum" also includes the relatively broad hydrogen lines present in both the $\mathrm{HZ}$ and $\mathrm{H}$ models. Because the continuum flux of an $\mathrm{HZ}$ model is larger than that of a comparable $\mathrm{H}$ model for $\lambda \gtrsim 200 \AA$, and the converse is true for shorter wavelengths, a heavy element will receive more (less) radiative support in an $\mathrm{HZ}$ model if its line opacity is more (less) important in the spectral range $\lambda \gtrsim 200 \AA$ than in the spectral region $\lambda \lesssim 200 \AA$.

\subsection{Other Improvements}

In an important paper, Gonzalez et al. (1995) recently discussed a number of improvements in the computations of radiative accelerations in stellar envelopes. It turns out that, along with the questions considered in $\S 2.1$ and $\S 2.2$ above, the last significant problem addressed in the context of equilibrium radiative levitation theory in white dwarfs is how to describe the momentum redistribution process following the absorption 


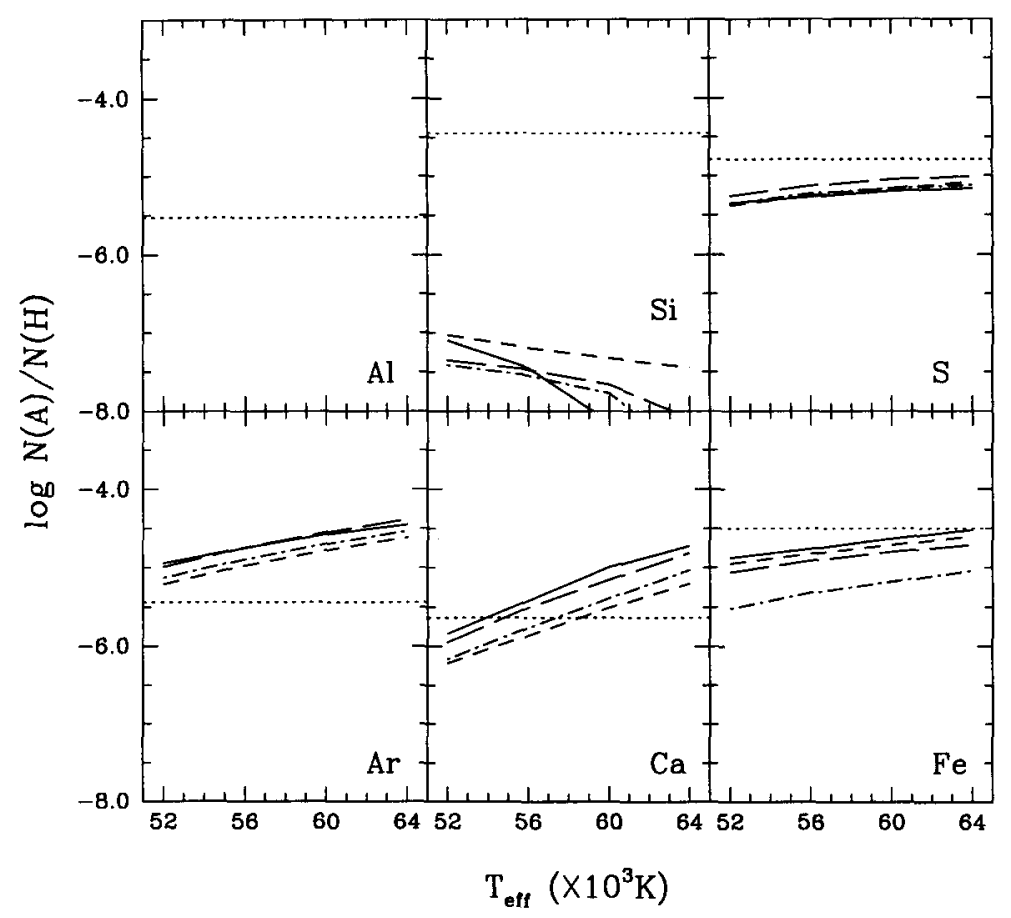

FIGURE 1. Continued

of a photon by an ion. In the method used by CFW, the probabilities that the newly gained momentum of the excited ion is spent either in its initial ionization state $i$ or in the higher state $i+1$ are assigned to the ion as a whole. In contrast, in the improved discussion of Gonzalez et al. (1995), such probabilities are assigned to each and every individual radiative transition considered in the calculation of the radiative acceleration. Moreover, Gonzalez et al. (1995) show that all previous studies (including the work of CFW) have underestimated the effects of electronic collisions on the ionization rates. As a result, the improved treatment of Gonzalez et al. (1995) leads to a momentum redistribution process that tends to favor the higher ionization state $i+1$. In contrast, most of the newly gained momentum is spent in state $i$ in the method used by CFW. Because the higher ionization state $i+1$ is less mobile than state $i$ (its higher charge leads to a larger interaction with the background plasma), the prescription of Gonzalez et al. (1995) leads to smaller radiative accelerations than the method used by CFW and many others before.

To follow up on the work of CFW, we present the results of additional calculations that incorporate the detailed recipe proposed by Gonzalez et al. (1995). Along with this improved prescription, our calculations also incorporate an upgraded value for the line profile width associated with pressure broadening. This new line profile width is also suggested by the work of Gonzalez et al. (1995). This change leads to a relatively small but systematic increase of the radiative accelerations and equilibrium abundances. Figure 1 compares the predicted surface equilibrium abundances of our twelve selected heavy elements calculated according to the above procedures (solid, dashed, and long- 
dashed curves) to those calculated according to the improved procedure just described (dot-dashed curves). In the latter case, the Eddington flux used in the radiative accelerations calculations was taken from our grid of $\mathrm{HZ}$ model atmospheres. We observe that the improved estimates of the equilibrium abundances are generally systematically smaller than before, indicating that the momentum redistribution process of Gonzalez et al. (1995) is the dominant new mechanism. A few exceptions arise for $\mathrm{Si}, \mathrm{S}, \mathrm{Ar}$, and Ca. Taking into account the contributing effects of increasing the line width (which leads to an increase of the equilibrium abundances), we find that the estimate of CFW, which suggests an overall decrease of the abundances by a factor $\sim 3$ because of the momentum redistribution process, is quite realistic.

\section{Conclusion}

In their concluding remarks, CFW suggest a number of avenues for improving the calculations of radiative accelerations in the context of equilibrium radiative levitation theory in hot white dwarfs. We have addressed these issues here, and the present effort can be considered to complement the work of CFW. Our "best" results are given by the dot-dashed curves in Figure 1. We again emphasize that these results can only be considered as illustrative because of the inconsistencies between the fixed chemical composition of the background plasma and the derived equilibrium abundances of the various heavy elements. They show, however, that the relative abundances of levitating elements can be significantly altered, particularly in the present examples, by the presence of an opaque element such as Fe.

This work is supported by NASA contract NAS5-30180.

\section{REFERENCES}

Bergeron, P., Wesemael, F., Michaud, G., \& Fontaine, G. 1988, ApJ, 332, 964

Chayen, P., \& VenNes, S. 1995, in preparation

Chayer, P., Fontaine, G., \& Wesemael, F. 1995, ApJ, in press

Chayer, P., LeBlanc, F., Fontane, G., Wesemael, F., Michaud, G., \& Vennes, S. 1994, ApJ, 436, L161

Gonzalez, J. -F., LeBlanc, F., Artru, M. -C., Michaud, G. 1995, A\&A, in press

Vennes, S., Pradhan, A. K., Thejll, P. 1995, in preparation

Vennes, S., Chayer, P., Thorstensen, J. R., Bowyer, S., \& Shipman, H. L. 1992, ApJ, 392, L27

Werner, K., \& Dreizler, S. 1994, A\&A, 286, L31

Wesemael, F., Henry, R. B. C., \& Shipman, H. L. 1984, ApJ, 287, 868 